\title{
High-Mobility Group Box-1 Induces Decreased Brain-Derived Neurotrophic Factor-Mediated Neuroprotection in the Diabetic Retina
}

\author{
Ahmed M. Abu El-Asrar, Mohd Imtiaz Nawaz, Mohammad Mairaj Siddiquei, \\ Abdullah S. Al-Kharashi, Dustan Kangave, and Ghulam Mohammad \\ Department of Ophthalmology, College of Medicine, King Saud University, King Abdulaziz University Hospital, Old Airport Road, \\ P.O. Box 245, Riyadh 11411, Saudi Arabia \\ Correspondence should be addressed to Ahmed M. Abu El-Asrar; abuasrar@ksu.edu.sa
}

Received 15 April 2013; Accepted 25 April 2013

Academic Editor: Katarzyna Zorena

Copyright (C) 2013 Ahmed M. Abu El-Asrar et al. This is an open access article distributed under the Creative Commons Attribution License, which permits unrestricted use, distribution, and reproduction in any medium, provided the original work is properly cited.

\begin{abstract}
To test the hypothesis that brain-derived neurotrophic factor-(BDNF-) mediated neuroprotection is reduced by high-mobility group box-1 (HMGB1) in diabetic retina, paired vitreous and serum samples from 46 proliferative diabetic retinopathy and 34 nondiabetic patients were assayed for BDNF, HMGB1, soluble receptor for advanced glycation end products (sRAGE), soluble intercellular adhesion molecule-1 (sICAM-1), monocyte chemoattractant protein-1 (MCP-1), and TBARS. We also examined retinas of diabetic and HMGB1 intravitreally injected rats. The effect of the HMGB1 inhibitor glycyrrhizin on diabetes-induced changes in retinal BDNF expressions was studied. Western blot, ELISA, and TBARS assays were used. BDNF was not detected in vitreous samples. BDNF levels were significantly lower in serum samples from diabetic patients compared with nondiabetics, whereas HMGB1, sRAGE, sICAM-1, and TBARS levels were significantly higher in diabetic serum samples. MCP-1 levels did not differ significantly. There was significant inverse correlation between serum levels of BDNF and HMGB1. Diabetes and intravitreal administration of HMGB1 induced significant upregulation of the expression of HMGB1, TBARS, and cleaved caspase-3, whereas the expression of BDNF and synaptophysin was significantly downregulated in rat retinas. Glycyrrhizin significantly attenuated diabetes-induced downregulation of BDNF. Our results suggest that HMGB1-induced downregulation of BDNF might be involved in pathogenesis of diabetic retinal neurodegeneration.
\end{abstract}

\section{Introduction}

Diabetic retinopathy, a vision-threatening disease, is classically regarded as microvasculopathy. However, recent evidence suggests that diabetic retinopathy is a progressive neurodegenerative disease in which visual dysfunction is initiated early after the onset of diabetes and progresses independently of the vascular lesions [1-4]. However, the molecular mechanisms underlying the diabetes-induced retinal neurodegeneration and dysfunction are still not well understood. Recent studies revealed that diabetic retinal neurodegeneration is associated with oxidative stress resulting from excess generation of reactive oxygen species as well as inflammation $[3,5]$.

Brain-derived neurotrophic factor (BDNF), a protein belonging to the neurotrophin family, is expressed in retinal ganglion cells and Müller cells [6] and is important for the survival of retinal ganglion cells [7]. BDNF is important in neural development and cell survival and is essential to molecular mechanisms of synaptic activity [8]. Recent studies suggested that the early retinal neuropathy of diabetes involves the reduced expression of BDNF and can be ameliorated by an exogenous supply of this neurotrophin $[1,3]$. It was also demonstrated that the reduction of BDNF in the diabetic retina was attenuated by the antioxidant lutein, indicating that this change was partly caused by excessive oxidative stress [3].

High-mobility group box-1 (HMGB1) is a nonhistone DNA-binding nuclear protein that is highly conserved during evolution. It stabilizes nucleosome formation and facilitates gene transcription. Necrotic cell death can result in passive leakage of HMGB1 from the cell as the protein 
is then no longer bound to DNA. In addition, HMGB1 can be actively secreted by different cell types, including activated monocytes and macrophages, mature dendritic cells, natural killer cells, and endothelial cells. Extracellular HMGB1 functions as a proinflammatory cytokine [912]. Released HMGB1 signals through the receptor for advanced glycation end products (RAGE), a member of the immunoglobulin superfamily of receptors, leading to activation of the transcription factor nuclear factor kappa $\mathrm{B}(\mathrm{NF}-\kappa \mathrm{B})$, which may alter gene transcription and lead to the upregulation of proinflammatory cytokines, chemokines, and adhesion molecules and intensifies cellular oxidative stress [9-13], processes that may play a role in the pathogenesis of diabetic retinal neurodegeneration and dysfunction. Therefore, recently, RAGE has been implicated in the pathogenesis of various diabetic complications, via oxidative stress $[13,14]$. The causal relationship between persistent neuroinflammation and neurodegenerative process is becoming increasingly recognized [15]. Strong evidence indicates that chronic, low-grade inflammation is implicated in the pathogenesis of diabetic retinopathy $[16,17]$. It was also demonstrated that HMGB1 provides the link between chronic neuroinflammation and progressive neurodegeneration in neurodegenerative diseases, such as Parkinson's disease [15]. In addition, it was reported that extracellularly released HMGB1 protein mediates postischemic damage of the brain and retina and that inhibiting or knockdown of HMGB1 attenuated postischemic neurodegeneration [1821].

In previous studies, we demonstrated that HMGB1 was upregulated in the vitreous fluid and epiretinal membranes from patients with proliferative diabetic retinopathy (PDR) as well as in the retinas of diabetic mice. In addition, we demonstrated significant positive correlations between levels of HMGB1 and levels of inflammatory biomarkers such as monocyte chemoattractant protein-1 (MCP-1) and soluble intercellular adhesion molecule-1 (sICAM-1) in vitreous fluid from patients with PDR [22-24]. Glycyrrhizin (GA), an ingredient of the licorice roots, has long been known to exhibit glucocorticoid-like anti-inflammatory actions by inhibiting $11 \beta$-hydroxysteroid dehydrogenase. More recently, GA has also been shown to bind to and inhibit cytokine-like activities of HMGB1 [25].

In this study, we explored the hypothesis that BDNFmediated neuroprotection is reduced by HMGB1 in the diabetic retina. To test this hypothesis, we measured the levels of BDNF, HMGB1, soluble RAGE (sRAGE), biomarkers of inflammation and endothelial dysfunction including MCP-1, and sICAM- 1 and the oxidative stress and lipid peroxidation marker thiobarbituric acid reactive substances (TBARS) in the vitreous fluid and serum from a series of patients with PDR. In addition, we investigated the expression of BDNF, HMGB1, the synaptic vesicles protein synaptophysin, TBARS, and the apoptosis executer enzyme cleaved caspase- 3 in the retinas of diabetic rats. We also examined the effect of intravitreal administration of HMGB1 on the retinas of normal rats and whether constant GA intake suppresses diabetes-induced changes in BDNF expression.

\section{Materials and Methods}

2.1. Vitreous and Paired Serum Samples Collection and Preparation. Undiluted vitreous fluid samples $(0.3-0.6 \mathrm{~mL})$ and paired serum samples were obtained from 46 patients with PDR during pars plana vitrectomy. The indications for vitrectomy were traction retinal detachment and/or nonclearing vitreous hemorrhage. The diabetic patients were 35 males and 11 females, whose ages ranged from 22 to 80 years with a mean of $53.9 \pm 12.8$ years. The duration of diabetes ranged from 7 to 32 years with a mean of $16.4 \pm 5.6$ years. Twentyfour patients had insulin-dependent diabetes mellitus, and 22 patients had noninsulin-dependent diabetes mellitus. At presentation, the fasting blood glucose was uncontrolled in 15 patients and controlled in 40 patients. Twenty-six patients were receiving treatment for hypertension. The control group consisted of 34 patients who had undergone vitrectomy for the treatment of rhegmatogenous retinal detachment (RD) with no proliferative vitreoretinopathy. Controls were free from systemic disease and were 23 males and 11 females whose ages ranged from 12 to 82 years with a mean of $47.8 \pm 16.8$ years. Vitreous samples were collected undiluted by manual suction into a syringe through the aspiration line of vitrectomy, before opening the infusion line. The samples were centrifuged (5000 rpm for $10 \mathrm{~min}, 4^{\circ} \mathrm{C}$ ) and the supernatants were aliquoted and frozen at $-80^{\circ} \mathrm{C}$ until assay. Blood was collected after an overnight fast, and serum was obtained by centrifugation and stored at $-70^{\circ} \mathrm{C}$. The study was conducted according to the tenets of the Declaration of Helsinki, and informed consent was obtained from all patients. The study was approved by the Research Centre, College of Medicine, King Saud University.

2.2. Animals. All procedures with animals were performed in accordance with the ARVO statement for use of animals in ophthalmic and vision research and were approved by the institutional animal care and use committee of the College of Pharmacy, King Saud University. Adult male Sprague Dawley rats, 8-9 weeks of age weighting in the range of 210-230 g, were overnight fasted and streptozotocin (STZ; $65 \mathrm{mg} / \mathrm{kg}$ in $10 \mathrm{mM}$ sodium citrate buffer, $\mathrm{pH}$ 4.5; Sigma, St. Louis, MO, USA) was injected intraperitoneally. Equal volumes of citrate buffer were injected in nondiabetic animals. Measuring of blood glucose concentrations and body weight was started after 3 days of STZ injection. Diabetes was confirmed by assaying glucose concentration in the blood taken from tail vein. Rats with glucose levels $>250 \mathrm{mg} / \mathrm{dL}$ were considered to have diabetes. After 4 weeks of diabetes, animals were anesthetized by intraperitoneal injection of an overdose of chloral hydrate and sacrificed by decapitation. Retinas were dissected out, snap frozen, and stored at $-80^{\circ} \mathrm{C}$ until use. Similarly, retinas were obtained from age-matched nondiabetic control rats.

2.3. Intravitreal Injection of HMGB1. Sprague Dawley rats (220-230 g) were kept under deep anesthesia, and sterilized solution of recombinant HMGB1 ( $5 \mathrm{ng} / 5 \mu \mathrm{L}$; R\&D Systems, Minneapolis, MN, USA) was injected into the vitreous of the 
right eye. For the control, the left eye received $5 \mu \mathrm{L}$ of sterile phosphate buffer saline (PBS). The animals were sacrificed 4 days after intravitreal administration, and the retinas were carefully dissected, snap frozen in liquid nitrogen, and stored at $-80^{\circ} \mathrm{C}$ until analyzed.

2.4. Glycyrrhizin Treatment. Sprague Dawley rats were made diabetic as previously described. Diabetic rats were divided into 2 groups: the rats in group I received normal drinking water without any supplementation, and group II received drinking water supplemented with glycyrrhizic acid (150 mg/kg/day, Santa Cruz Biotechnology, Inc., Santa Cruz, CA, USA) immediately after establishment of diabetes. Each group consisted of 8-12 rats. After 4 weeks of diabetes, the rats were sacrificed, the eyes were removed, and retinas were isolated and frozen immediately in liquid nitrogen and stored at $-80^{\circ} \mathrm{C}$ until analyzed.

2.5. Enzyme-Linked Immunosorbent Assay (ELISA) Kits. ELISA kits for human BDNF (Quantikine Brain-Derived Neurotrophic factors Factor, Cat. no. DBD00), human MCP-1 (Quantikine Human Monocytes Chemotactic Protein-1, Cat. no. DCP00), human sRAGE (Quantikine Human Receptor for Advance glycation End products, Cat. no. DRG00) and human sICAM-1 (Quantikine Human Soluble Intercellular Adhesion Molecules-1, Cat. no. DCD540) were purchased from R\&D Systems. An ELISA kit for HMGB1 (human high-mobility group box-1, Cat. no. ST51011) was purchased from IBL International GMBH (Hamburg, Germany). The detection limits for BDNF, MCP-1, sRAGE, sICAM, and HMGB1 were 20, 5, 4.12, 96, and 200 picograms $/ \mathrm{mL}$ (pg/mL), respectively. The ELISA plate readings were done using FLUOstar Omega-Microplate reader from BMG Labtech, Offenburg, Germany.

2.6. Oxidative Stress Marker Assay Kit. The assay kit for the oxidative stress and lipid peroxidation marker TBARS (Cat. no. 10009055) was purchased from Cayman Chemical Company, Ann Arbor, MI, USA.

2.7. Measurement of BDNF, MCP-1, sRAGE, sICAM, and HMGB1 in Human Vitreous and Serum and BDNF in Rat Retinas. The quantifications of the level of BDNF, MCP-1, sRAGE, sICAM, and HMGB1 in the vitreous and serum and in the rat retinas were determined using specific ELISA kits according to the manufacturer's instruction. For each ELISA kit, the undiluted standard served as the highest concentration and calibrator diluents served as the blank. Depending upon the detection range of the ELISA kit and the expression level of the particular molecule, vitreous and serum samples were either directly used or diluted with calibrator diluent supplied with ELISA kit.

For measurement of BDNF in the vitreous, $50 \mu \mathrm{L}$ of undiluted samples was added to ELISA plates for analysis. For serum, samples were diluted 25-fold, 5-fold, 2-fold, and 20fold for BDNF, MCP-1, sRAGE, and sICAM-1 measurements, respectively. $100 \mu \mathrm{L}, 200 \mu \mathrm{L}, 50 \mu \mathrm{L}$, and $100 \mu \mathrm{L}$ of diluted sample for BDNF, MCP-1, sRAGE and sICAM were added into each of the ELISA plates for the analysis. For measurement of BDNF in rat retinas, $200 \mu \mathrm{g}$ of rat retinal homogenate was used and added into each of the ELISA plates for the analysis. For the quantification of HMGB1 within the high sensitivity range, $50 \mu \mathrm{L}$ of diluents buffer (Dilbuf, IBL International) was added to each well of the plate followed by the addition of $50 \mu \mathrm{L}$ of 2 -fold diluted sample. Following sample incubation into the wells of ELISA plates, secondary antibodies against BDNF, MCP-1, sRAGE, sICAM and HMGB1 conjugated to horseradish peroxidase were added to each well of the ELISA plate. After incubation, substrate mix solution was added for color development. The reaction was stopped by the addition of $2 \mathrm{~N}$ sulfuric acid and optical density (OD) was read at $450 \mathrm{~nm}$ in microplate reader. Each assay was performed in duplicate. Using the 4-parameter fit logistic (4$\mathrm{PL}$ ) curve equation, the actual concentration for each sample was calculated. For the samples that have been diluted, the correction read from the standard curve obtained using 4-PL was multiplied by the dilution factors to get the actual reading for each sample.

2.8. Measurement of TBARS in Human Serum and Rat Retinas. The steps for the measurement of TBARS in serum and in rat retinal homogenate were followed as per the manufacturer instructions that use the principle of formation of adduct between malondialdehyde (MDA) and thiobarbituric acid (TBA) under high temperature $\left(95^{\circ} \mathrm{C}\right)$ and acidic condition and color developed is measured colorimetrically. Color reagent to be used was prepared by mixing TBA with TBA acetic acid and TBA sodium hydroxide (supplied with the kit). In a $10 \mathrm{~mL}$ tube, $100 \mu \mathrm{L}$ undiluted serum and retinal homogenate were mixed with $100 \mu \mathrm{L}$ of sodium dodecyl sulphate (SDS). The color reagent $(4 \mathrm{~mL})$ was added in each respective tube and was boiled for 1 hour. Tubes were cooled immediately on ice for 10 minutes and were centrifuged for 10 minutes at $1600 \times \mathrm{g}$ at $4^{\circ} \mathrm{C}$. The upper clear solution was loaded on 96-well clear plates and the color was measured at $530 \mathrm{~nm}$ using FLUOstar Omega-Microplate reader (BMG Labtech).

2.9. Western Blot Analysis. Retinas were homogenized in a Western lysis buffer (30 mM Tris-HCL; pH 7.5, 5 mM EDTA, $1 \%$ Triton X-100, $250 \mathrm{mM}$ sucrose, $1 \mathrm{mM}$ sodium vanadate, and protease inhibitor cocktail). The protease inhibitor used was "Complete without EDTA" (Roche, Mannheim, Germany). The lysate was centrifuged at $14,000 \times \mathrm{g}$ for $10 \mathrm{~min}$ at $4^{\circ} \mathrm{C}$, and the supernatant was collected. Protein content was assayed by DC protein assay (Bio-Rad Laboratories, Hercules, CA, USA). The tissue lysateS containing $50 \mu \mathrm{g}$ protein were separated on 10-15\% SDS-polyacrylamide gels and were transferred onto polyvinylidene difluoride (PVDF) membranes. The blots were blocked with TBST (20 mM Tris$\mathrm{HCl}$; pH 7.6, $136 \mathrm{mM} \mathrm{NaCl}$, and 0.1\% Tween-20) containing $5 \%$ nonfat milk.

For detection of BDNF, synaptophysin, cleaved caspase3, and HMGB1, the membrane was incubated overnight at $4^{\circ} \mathrm{C}$ with BDNF mouse monoclonal anti-BDNF $(1: 500$, 
TABLE 1: Comparisons of mean levels for brain-derived neurotrophic factor (BDNF), high-mobility group box-1 (HMGB1), monocyte chemoattractant protein-1 (MCP-1), soluble intercellular adhesion molecule-1 (sICAM-1), soluble receptor for advanced glycation end products (sRAGE), and thiobarbituric acid reactive substance (TBARS) in serum samples.

\begin{tabular}{lcccccc}
\hline Disease group & $\begin{array}{c}\text { BDNF } \\
(\mathrm{ng} / \mathrm{mL})\end{array}$ & $\begin{array}{c}\text { HMGB1 } \\
(\mathrm{mg} / \mathrm{mL})\end{array}$ & $\begin{array}{c}\text { MCP-1 } \\
(\mathrm{pg} / \mathrm{mL}) \\
(\mathrm{mean} \pm \mathrm{s} . \mathrm{d})\end{array}$ & $\begin{array}{c}\text { sICAM } \pm \mathrm{s} . \mathrm{d}) \\
(\mathrm{ng} / \mathrm{mL})\end{array}$ & $\begin{array}{c}\text { sRAGE }) \\
(\mathrm{pg} / \mathrm{mL})\end{array}$ & $\begin{array}{c}\text { TBARS } \\
(\mu \mathrm{M})\end{array}$ \\
\hline PDR & $10.2 \pm 7.7$ & $4.4 \pm 2.1$ & $267.34 \pm 174.0$ & $205.2 \pm 99.1$ & $898.1 \pm 536.9$ & $29.0 \pm 20.7$ \\
RD & $16.7 \pm 10.3$ & $2.2 \pm 1.4$ & $267.29 \pm 187.7$ & $157.0 \pm 45.7$ & $589.7 \pm 320.9$ & $17.2 \pm 6.1$ \\
$\begin{array}{l}\text { Mean comparison } \\
P \text {-value }\end{array}$ & $0.015^{*}$ & $<0.001^{*}$ & 0.836 & $0.019^{*}$ & $0.008^{*}$ & $0.011^{*}$ \\
\hline
\end{tabular}

${ }^{*}$ Statistically significant at $5 \%$ level of significance.

PDR: proliferative diabetic retinopathy; RD: rhegmatogenous retinal detachment.

Cat no. SC-65513, Santa Cruz), goat polyclonal antisynaptophysin (1 $\mu \mathrm{g} / \mathrm{mL}$, Cat. no. AF-5555, R\&D Systems), rabbit monoclonal anticleaved caspase-3 (1:300, Cat. no. MAB835, R\&D Systems) and rabbit polyclonal anti-HMGB1 (1:1000, Cat. no. ab18256, Abcam). After overnight incubation with primary antibodies, the membranes were washed four times with TBS-T (5 min each). For BDNF, the membrane was incubated at room temperature for $1.5 \mathrm{~h}$ with antimouse secondary horseradish peroxidase-conjugated antibody (1:2000, SC-2005, Santa Cruz), for synaptophysin with anti-goat secondary horseradish peroxidase-conjugated antibody (1:2000, SC-2768, Santa Cruz), and for cleaved caspase3 and HMGB1 with anti-rabbit secondary horseradish peroxidase-conjugated antibody $(1: 2000$, SC-2004, Santa Cruz). After incubations with secondary antibodies, membranes were washed four times with TBS-T ( 5 min each) and the immunoreactivity of bands was visualized on a highperformance chemiluminescence machine (G: Box ChemiXX8 from Syngene, Synoptic Ltd. Cambridge, UK) by using enhanced chemiluminescence plus Luminol (sc-2048, Santa Cruz) and quantified by densitometric analysis using image processing and analysis in GeneTools (Syngene by Synoptic Ltd. Cambridge, UK). For loading control, membranes were stripped and incubated with a mouse monoclonal anti- $\beta$ actin antibody $(1: 2000$, SC-2048, Santa Cruz) and all the remaining steps were followed as detailed above. All data from the three independent experiments were expressed as a ratio to mean intensity.

2.10. Statistical Analysis. The nonparametric Mann-Whitney $U$ test was used to compare means from two independent study groups. Pearson correlation coefficients were computed to investigate correlations between continuous variables. A $P$ value less than 0.05 indicated statistical significance. SPSS version 15.0 program was used for the statistical analysis.

\section{Results}

3.1. Levels of BDNF, HMGB1, sRAGE, sICAM-1, MCP-1, and TBARS in Patients with PDR and Nondiabetic Control Subjects. BDNF was not detected in vitreous samples from patients with PDR and nondiabetic control patients. BDNF, HMGB1, sRAGE, sICAM-1, MCP-1, and TBARS were detected in all serum samples from patients with PDR and nondiabetic controls. Mean levels of BDNF in serum samples from patients with PDR were significantly lower than those in nondiabetic control patients $(P=0.015)$. On the other hand, mean levels of HMGB1, sRAGE, sICAM-1, and TBARS were significantly higher in serum samples from patients with PDR than those in nondiabetic controls $(P<0.001 ; P=$ $0.008 ; P=0.019 ; P=0.011$, resp.). Mean levels of MCP1 did not differ significantly between patients with PDR and nondiabetic control patients $(P=0.836)$ (Table 1$)$.

3.2. Correlations. There was a significant inverse correlation between serum levels of BDNF and HMGB1 $(r=-0.324 ; P=$ 0.049 ) (Figure 1). There were significant positive correlations between serum levels of TBARS and sRAGE $(r=0.335 ; P=$ $0.018)$, sICAM-1 $(r=0.303 ; P=0.032)$, and MCP-1 $(r=$ $0.344 ; P=0.012)$. There was a significant positive correlation between serum levels of sRAGE and sICAM-1 $(r=0.431$; $P<0.001$ ) (Table 2).

3.3. Relationships between Serum BDNF, HMGB1, sRAGE, sICAM-1, MCP-1, and TBARS Levels and Clinical Variables at Presentation in Patients with PDR. We examined the correlations between serum BDNF, HMGB1, sRAGE, sICAM1, MCP-1, and TBARS levels and clinical parameters at presentation including fasting blood sugar, triglycerides, total cholesterol, HDL-cholesterol, LDL-cholesterol, and creatinine. There were significant positive correlations between serum levels of MCP-1, and triglycerides ( $r=0.402 ; P=$ $0.0231)$ and total cholesterol $(r=0.519 ; P=0.003)$. No other significant correlations were detected.

There were no significant relationships between serum levels of BDNF, HMGB1, sRAGE, sICAM-1, MCP-1 and TBARS and type of diabetes treatment (Table 3 ). The mean serum levels of sRAGE in patients receiving antihypertensive agents were significantly higher than those in other patients (Table 4).

3.4. Severity of Hyperglycemia in Rats. The body weights of the rats with diabetes were lower and their blood glucose levels were more than fourfold higher compared with agematched normal control rats $(180 \pm 22$ versus $250 \pm 28 \mathrm{~g}$ and $453 \pm 32$ versus $111 \pm 12 \mathrm{mg} / \mathrm{dL}$, resp.). Treatment of the diabetic rats with GA for one month did not change these 
TABLE 2: Pearson correlation coefficients between serum levels of the studied factors.

\begin{tabular}{lcccc}
\hline & BNDF & HMGB1 & MCP-1 & TBARS \\
\hline \multirow{2}{*}{ HMGB1 } & $r=-0.324$ & & & \\
& $P=0.049^{*}$ & & & \\
MCP-1 & $r=0.089$ & -0.156 & & \\
& $P=0.519$ & 0.250 & 0.344 & \\
TBARS & $r=-0.115$ & 0.121 & $0.012^{*}$ & 0.303 \\
& $P=0.413$ & 0.382 & 0.168 & $0.032^{*}$ \\
sICAM & $r=-0.103$ & -0.016 & 0.219 & $0.335<$ \\
sRAGE & $P=0.450$ & 0.907 & 0.241 & $0.018^{*}$ \\
\end{tabular}

${ }^{*}$ Statistically significant at $5 \%$ level of significance.

Where the row and column meet is the correlation coefficient and the $P$ value for the two variables.

BDNF: brain-derived neurotrophic factor; HMGB1: high-mobility group box-1; MCP-1: monocyte chemoattractant protein-1; TBARS: thiobarbituric acid reactive substance; sICAM-1: soluble intercellular adhesion molecule-1; sRAGE: soluble receptor for advanced glycation end products.

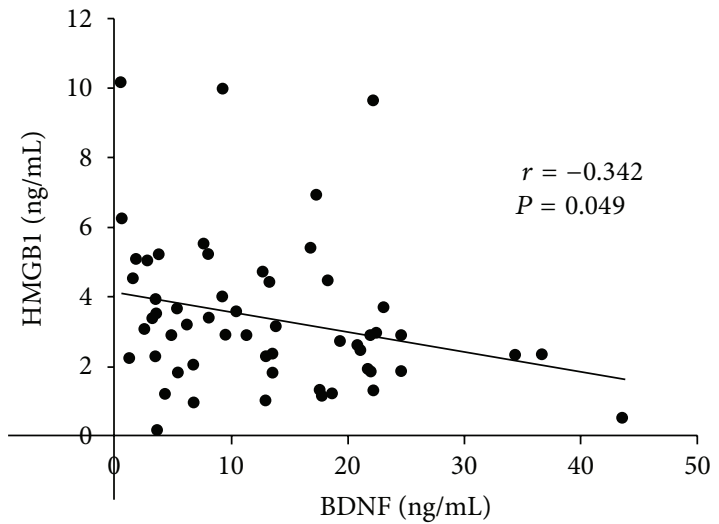

FIGURE 1: Correlation between serum levels of high-mobility group box-1 (HMGB1) and brain-derived neurotrophic factor (BDNF). There is a significant inverse correlation between serum levels of HMGB1 and BDNF.

metabolic variables in diabetic rats $(167 \pm 25$ versus $178 \pm 22 \mathrm{~g}$ and $449 \pm 36$ versus $475 \pm 32 \mathrm{mg} / \mathrm{dL}$, resp.).

3.5. Effect of Hyperglycemia on the Expression of BDNF, HMGB1, TBARS, Synaptophysin, and Cleaved Caspase-3 in Rat Retinas. Quantification of BDNF levels in nondiabetic controls and the retinas of rats with diabetes was done with the use of ELISA. BDNF protein levels in the retinas of animals with diabetes $(0.03 \pm 0.01 \mathrm{pg} / \mu \mathrm{g}$ protein $)$ were significantly lower than those in nondiabetic controls $(0.06 \pm$ $0.02 \mathrm{pg} / \mu \mathrm{g}$ protein) $(P=0.014$; Mann-Whitney $U$ test) (Figure 2$)$. The generation of TBARS in diabetic retinas $(11.88 \pm$ $8.2 \mu \mathrm{mole} / \mu \mathrm{g}$ protein) significantly increased compared with nondiabetic controls $(5.27 \pm 1.8 \mu \mathrm{mole} / \mu \mathrm{g}$ protein $)(P=$ 0.005) (Figure 3). We quantified the expression of HMGB1, BDNF, synaptophysin, and cleaved caspase- 3 by Western blot analysis. Densitometric analysis of the bands revealed a significant increase in HMGB1 $(P=0.01)$ and cleaved caspase-3 $(P=0.004)$ and a significant decrease in BDNF
TABLE 3: Relationship between serum levels of the studied factors and type of diabetes treatment in patients with proliferative diabetic retinopathy.

\begin{tabular}{lccc}
\hline & \multicolumn{2}{c}{ Use of insulin } & Y value \\
& $\begin{array}{c}\text { Yes } \\
(\text { mean } \pm \text { s.d })\end{array}$ & $\begin{array}{c}\text { No } \\
(\text { mean } \pm \text { s.d })\end{array}$ & \\
\hline BDNF $(\mathrm{ng} / \mathrm{mL})$ & $10.1 \pm 8.4$ & $10.3 \pm 7.2$ & 0.749 \\
HMGB1 & $4.7 \pm 2.4$ & $4.2 \pm 1.9$ & 0.546 \\
$(\mathrm{ng} / \mathrm{mL})$ & $271.8 \pm 198.7$ & $263.4 \pm 155.2$ & 0.835 \\
MCP-1 $(\mathrm{pg} / \mathrm{mL})$ & $31.6 \pm 25.0$ & $26.9 \pm 16.9$ & 0.842 \\
TBARS $(\mu \mathrm{M})$ & $196.0 \pm 83.9$ & $214.5 \pm 113.4$ & 0.673 \\
sICAM-1 & & & \\
$(\mathrm{ng} / \mathrm{mL})$ & $1013.6 \pm 565.3$ & $787.9 \pm 496.1$ & 0.206 \\
sRAGE $(\mathrm{pg} / \mathrm{mL})$ &
\end{tabular}

${ }^{*}$ Statistically significant at $5 \%$ level of significance.

BDNF: brain-derived neurotrophic factor; HMGB1: high-mobility group box-1; MCP-1: monocyte chemoattractant protein-1; TBARS: thiobarbituric acid reactive substance; sICAM-1: soluble intercellular adhesion molecule-1; sRAGE: soluble receptor for advanced glycation end products.

$(P=0.046)$ and synaptophysin $(P=0.003)$ in diabetic retinas compared with nondiabetic controls (Figure 4$)$.

3.6. Effect of Intravitreal Administration of $H M G B 1$ on the Expression of BDNF, HMGB1, TBARS, Synaptophysin, and Cleaved Caspase-3 in Rat Retinas. ELISA demonstrated that intravitreal administration of HMGB1 in normal rats induced significant downregulation of the expression of BDNF in the retinas $(0.038 \pm 0.01 \mathrm{pg} / \mu \mathrm{g}$ protein $)$ compared with controls $(0.05 \pm 0.01 \mathrm{pg} / \mu \mathrm{g}$ protein $)(P=0.01)$ (Figure 2$)$. HMGB1 injection induced significant upregulation of the generation of TBARS in the retinas $(8.24 \pm 1.5 \mu \mathrm{mole} / \mu \mathrm{g}$ protein $)$ compared with controls $(5.51 \pm 1.9 \mu$ mole $/ \mu$ g protein $)(P=$ 0.045) (Figure 3). Western blot analysis for HMGB1, BDNF, synaptophysin, and cleaved caspase- 3 showed that intravitreal administration of HMGB1 in normal rats significantly increased the expression of HMGB1 $(P=0.001)$ and cleaved caspase-3 $(P=0.004)$ and significantly decreased 
TABLE 4: Relationship between the serum levels of the studied factors and presence or absence of hypertension in patients with proliferative diabetic retinopathy.

\begin{tabular}{lccc}
\hline & \multicolumn{2}{c}{ Presence of hypertension } & P value \\
& $\begin{array}{c}\text { Yes } \\
\text { (mean } \pm \text { s.d) }\end{array}$ & $\begin{array}{c}\text { No } \\
\text { (mean } \pm \text { s.d) }\end{array}$ & \\
\hline BDNF $(\mathrm{ng} / \mathrm{mL})$ & $10.1 \pm 7.7$ & $10.3 \pm 8.0$ & 0.907 \\
HMGB1 & $4.4 \pm 2.3$ & $4.5 \pm 1.8$ & 0.565 \\
$(\mathrm{ng} / \mathrm{mL})$ & $244.9 \pm 156.0$ & $300.2 \pm 199.4$ & 0.489 \\
MCP-1 $(\mathrm{pg} / \mathrm{mL})$ & $24.2 \pm 11.2$ & $35.7 \pm 28.5$ & 0.356 \\
TBARS $(\mu \mathrm{M})$ & $213.0 \pm 118.9$ & $194.1 \pm 61.8$ & 0.756 \\
sICAM-1 & $1050.5 \pm$ & $686.5 \pm$ & $0.017^{*}$ \\
(ng/mL) & 536.2 & 474.2 & \\
sRAGE $(\mathrm{pg} / \mathrm{mL})$ &
\end{tabular}

${ }^{*}$ Statistically significant at $5 \%$ level of significance.

BDNF: brain-derived neurotrophic factor; HMGB1: high-mobility group box-1; MCP-1: monocyte chemoattractant protein-1; TBARS: thiobarbituric acid reactive substance; sICAM-1: soluble intercellular adhesion molecule-1; sRAGE: soluble receptor for advanced glycation end products.

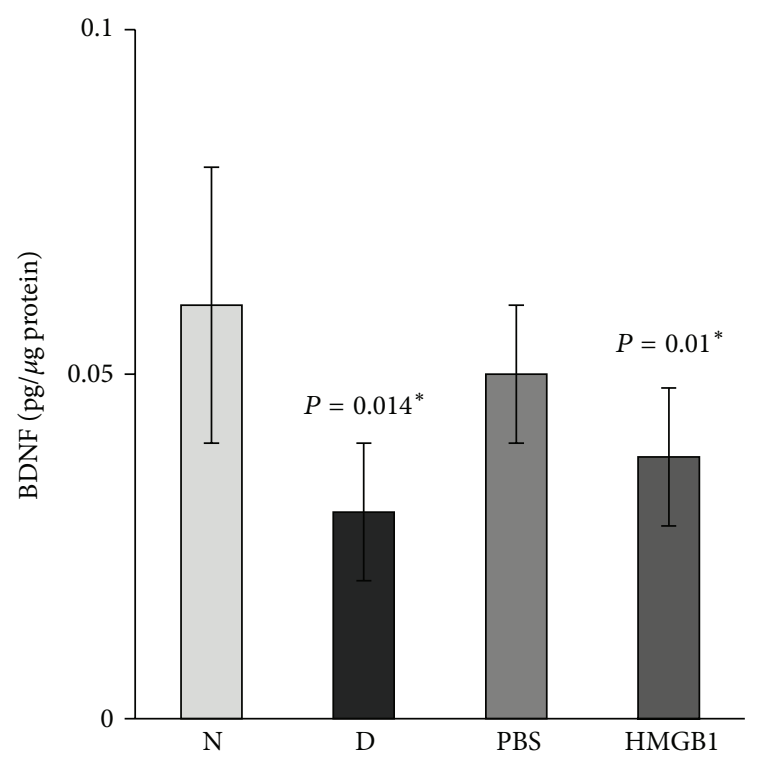

FIGURE 2: Enzyme-linked immunosorbent assay for brain-derived neurotrophic factor (BDNF) in rat retinas. There is a significant decrease in the expression of BDNF in the retinas of diabetic rats (D) compared with the nondiabetic control rats (N). Intravitreal administration of high-mobility group box-1 (HMGB1) induced a significant downregulation of the expression of BDNF compared with intravitreal administration of phosphate buffer saline (PBS). Each experiment was repeated 2 to 3 times with fresh samples $(n=$ $6)$.

the expression of BDNF $(P=0.001)$ and synaptophysin $(P=0.001)$ compared with controls (Figure 5).

3.7. HMGB1 Inhibitor Glycyrrhizin Attenuates the Effect of Diabetes. Western blot analysis was used to assess the effect of GA on diabetes-induced alterations of BDNF in the retinas of rats. Constant GA intake from the onset of diabetes

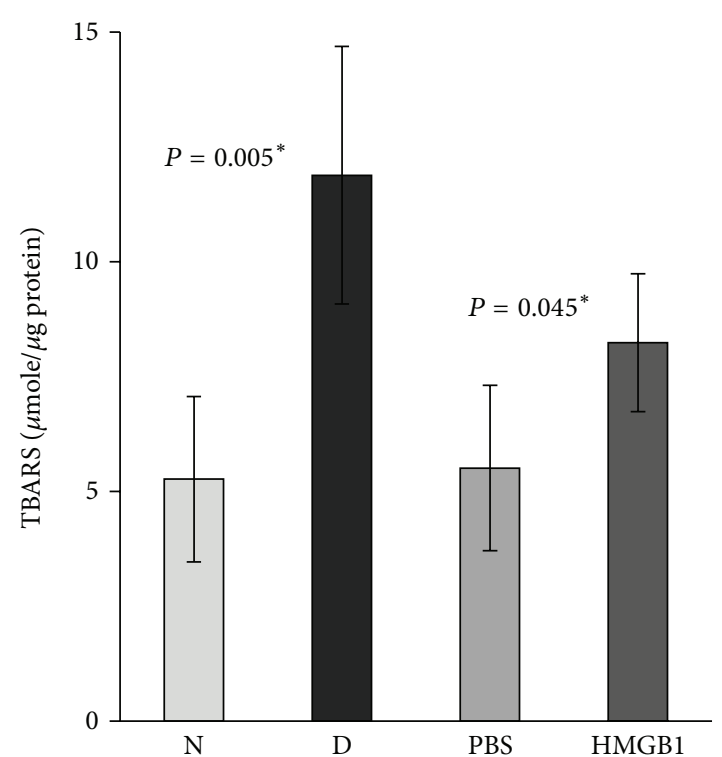

FIGURE 3: Levels of thiobarbituric acid reactive substances (TBARS) are significantly higher in the retinas of diabetic rats (D) compared with the nondiabetic control rats $(\mathrm{N})$. Intravitreal administration of high-mobility group box-1 (HMGB1) induced a significant upregulation of the levels of TBARS compared with intravitreal administration of phosphate buffer saline (PBS). Each experiment was repeated 2 to 3 times with fresh samples $(n=6)$.

significantly attenuated diabetes-induced downregulation of $\operatorname{BDNF}(P=0.048)$ (Figure 6). In a previous study, we demonstrated that constant GA intake from the onset of diabetes significantly attenuated diabetes-induced upregulation of HMGB1 in the retinas of rats [26].

\section{Discussion}

In the present study, we investigated the correlations between the levels of BDNF and the levels of HMGB1 in the vitreous fluid and serum from patients with PDR and in the retinas of rats with diabetes. We also investigated the effect of intravitreal administration of HMGB1 on the retinas of rats. We demonstrated that BDNF levels were below the detection limit of our test system in the vitreous fluid. BDNF was significantly downregulated in the serum from patients with PDR and in the retinas of rats with diabetes, whereas HMGB1 was significantly upregulated. We also found a significant inverse correlation between the levels of BDNF and HMGB1 in the serum. Intravitreal administration of HMGB1 to normal rats induced a significant downregulation of BDNF. Our results are consistent with previous reports that demonstrated reduced levels of the protein and mRNA of BDNF in streptozotocin-induced diabetic rat retinas [1, 3]. Sasaki et al. [3] reported that excessive oxidative stress is responsible for the reduced BDNF levels in the retinas from rats with diabetes. The antioxidant lutein prevented reactive oxygen species generation, visual impairment, BDNF depletion, and neuronal cell apoptosis in the diabetic retina. Furthermore, intraocular administration of BDNF rescued 


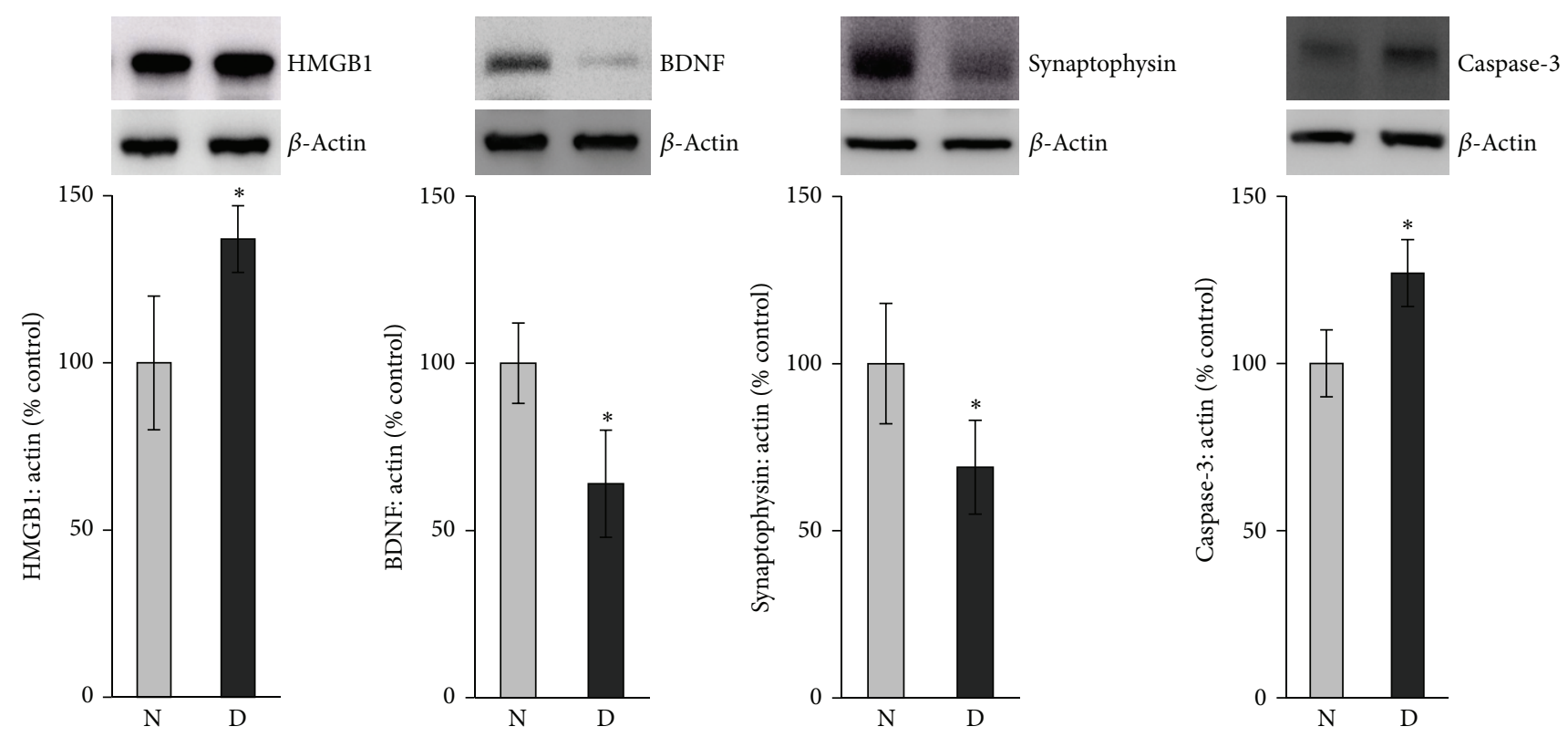

FIGURE 4: Western blot analysis of high-mobility group box-1 (HMGB1), brain-derived neurotrophic factor (BDNF), synaptophysin, and cleaved caspase- 3 in rat retinas. There is a significant increase in the expression of HMGB1 and cleaved caspase-3 and a significant decrease in the expression of BDNF and synaptophysin in the retinas of diabetic rats (D) compared with the nondiabetic control rats $(\mathrm{N})$. Each experiment was repeated 2 to 3 times with fresh samples $(n=6)$.
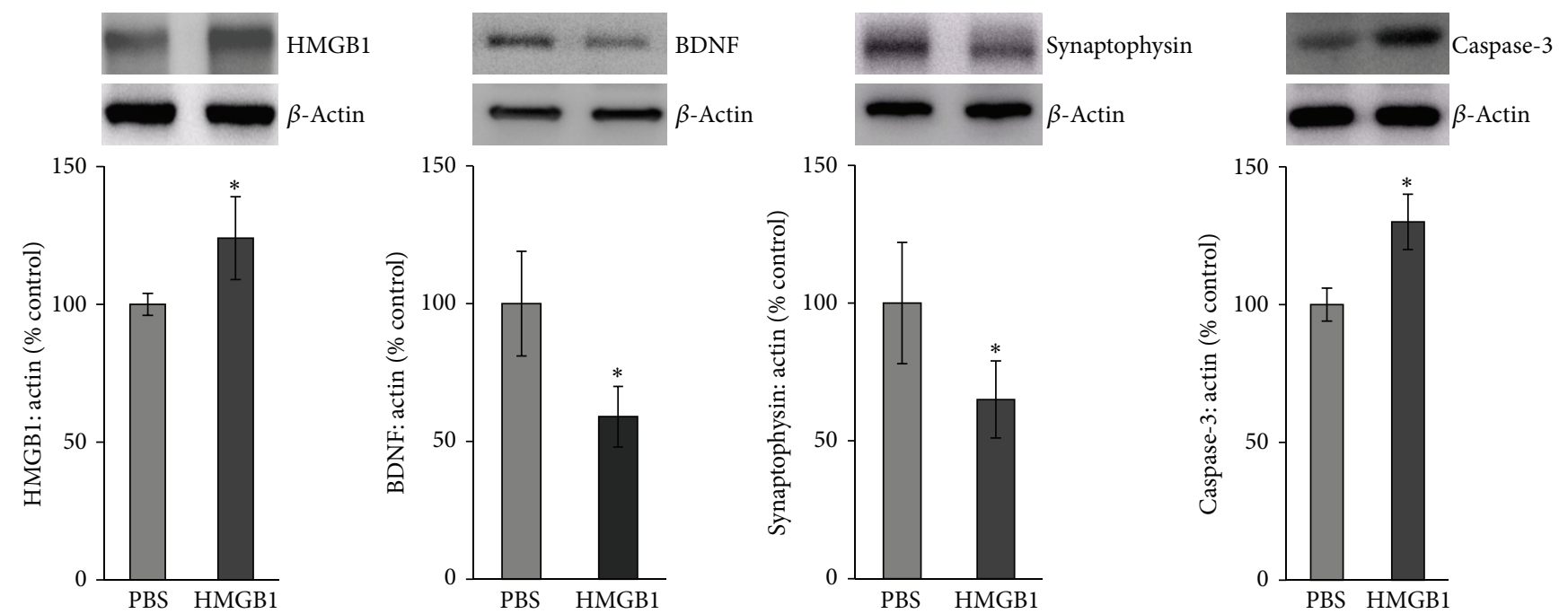

FIGURE 5: Western blot analysis of rat retinas. Intravitreal administration of high-mobility group box-1 (HMGB1) induced a significant upregulation of the expression of HMGB1 and cleaved caspase-3 and a significant downregulation of the expression of brain-derived neurotrophic factor (BDNF) and synaptophysin compared with intravitreal administration of phosphate buffer saline (PBS). Each experiment was repeated 2 to 3 times with fresh samples $(n=6)$.

retinal dopaminergic amacrine cells from neurodegeneration in rats with diabetes [1]. The findings suggest that the early retinal neuropathy of diabetes involves the reduced expression of BDNF, whose deficiency is associated with a number of neurodegenerative disorders [27]. We showed that the protein level of BDNF was also reduced in the retinas by intravitreal injection of HMGB1, whose levels are elevated in the vitreous fluid and epiretinal membranes from patients with PDR as well as in the retinas of diabetic animals [22-24].
In addition, the HMGB1 inhibitor GA attenuated diabetesinduced upregulation of HMGB1 and downregulation of BDNF in the retinas of rats. Our findings suggest that diabetes-induced decrease of BDNF in the retina seems to be mediated by HMGB1.

BDNF, in addition to its role in neuronal health, plays a systemic role in glucose metabolism. In animals, BDNF is involved in insulin resistance, reduces food intake, and lowers blood glucose levels in obese diabetic mice [28]. It was 


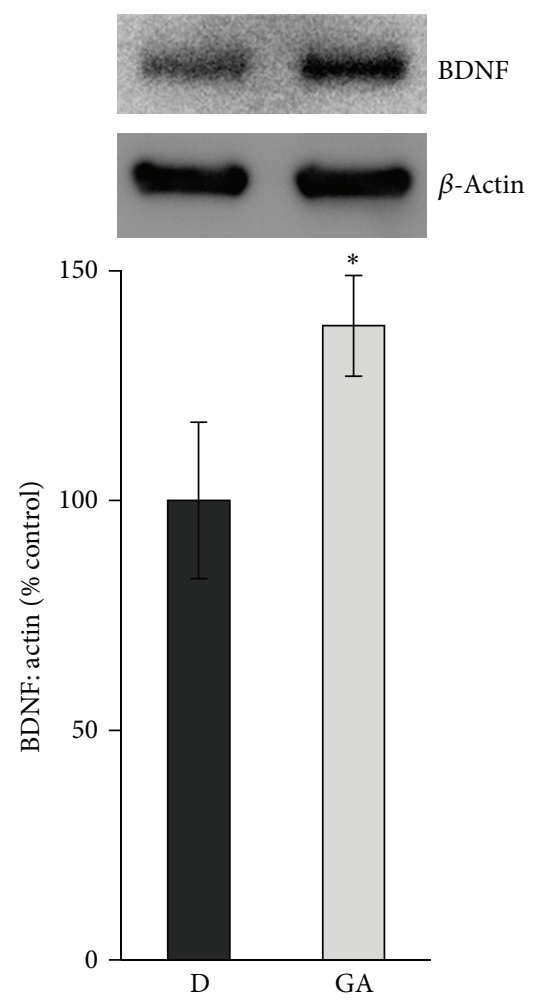

FIGURE 6: Diabetes-induced downregulation of brain-derived neurotrophic factor (BDNF) is prevented by constant intake of glycyrrhizic acid (GA) as shown by Western blot analysis. Each experiment was repeated 2 to 3 times with fresh samples $(n=6)$.

also demonstrated that, compared with thiazolidinediones, BDNF potently ameliorates pancreatic dysfunction, fatty liver, and energy expenditure, thereby exerting favourable antidiabetic effects in type 2 diabetic mice [29]. It was also reported that high levels of glucose, but not insulin, inhibit the output of BDNF from the human brain [30]. These findings suggest that BDNF may have a potential as a unique hypoglycemic agent for the treatment of diabetes. In line with earlier reports $[30,31]$, serum levels of BDNF in patients with PDR were significantly reduced compared to control nondiabetic patients. In contrast to BDNF, we demonstrated elevated levels of HMGB1 in the serum from patients with PDR. Our results are consistent with previous reports that demonstrated elevated levels of HMGB1 in the serum from patients with type 1 and type 2 diabetes $[32,33]$ and that higher serum HMGB1 levels were associated with greater prevalence and severity of albuminuria. In addition, HMGB1 levels were positively associated with markers of low-grade inflammation and endothelial dysfunction [33].

Synaptophysin is an integral membrane protein of the synaptic vesicles. It possibly serves multiple functions in synaptic vesicle formation and exocytosis, playing an important role in neurotransmitter delivery. It is widely used as one of the synaptic function markers and is also thought to be closely related to synaptogenesis and synaptic plasticity during neural tissue development. Synaptophysin knockout mice exhibited a significant decrease in synaptic vesicles in retinal rod photoreceptors, which disturbs neurotransmitter release and synaptic network activity [34]. Previous studies demonstrated that 1 month of diabetes decreases the retinal expression of synaptophysin $[3,35,36]$ and that the antioxidant lutein prevented synaptophysin reduction and avoided increase in cleaved caspase-3 in the diabetic retina [3] suggesting that local oxidative stress has a neurodegenerative influence in diabetic retina. In the present study, we demonstrated that, similar to diabetes, HMGB1 caused a significant decrease in the synaptic vesicle protein synaptophysin and a significant increase in the activated cleaved caspase- 3 in the retina of normal rats.

Activation of HMGB1/RAGE signaling axis is important in promoting proinflammatory pathways considered to play an important role in diabetes-induced retinal neuroinflammation $[9-12,16,17]$. Interaction of HMGB1 with RAGE results in activation of NF- $\kappa \mathrm{B}$, release of cytokines and chemokines, expression of adhesion molecules, and induction of oxidative stress [9-14]. In our laboratory, we recently demonstrated that diabetes induced significant upregulation of the expression of HMGB1, RAGE, activated NF- $\kappa$ B, and intercellular adhesion molecule-1 (ICAM-1) in the retinas of rats and that intravitreal administration of HMGB1 in normal rats mimics the effect of diabetes. In addition, coimmunoprecipitation studies showed that diabetes increases the interaction between HMGB1 and RAGE [26]. These findings suggest a pathogenic role of HMGB1 in the development of diabetic retinopathy through RAGE and activation of NF$\kappa \mathrm{B}$. Recently, it was reported that chronic neuroinflammation may be a driving force of progressive neurodegeneration and that HMGB1 provides the link between chronic neuroinflammation and progressive neurodegeneration in neurodegenerative diseases, such as Parkinson's disease [15].

In the present study, we report that sRAGE and sICAM1 levels were significantly upregulated in the serum from patients with PDR and that MCP-1 levels did not differ between patients with PDR and nondiabetic control subjects. Our results are consistent with previous reports that demonstrated increased circulating levels of sRAGE [32, 37, $38]$ and sICAM-1 $[32,39-41]$ in both patients with type 1 and type 2 diabetes mellitus compared to nondiabetic controls. Pham et al. [41] demonstrated increased serum concentration of sICAM- 1 in patients with type 2 diabetes mellitus compared with healthy subjects, whereas serum concentrations of the chemokine MCP-1 were not increased. It has been suggested that human sRAGE production might be derived from alternative RNA splicing as well as by release from the full-length RAGE receptor by proteinases [42]. It was also demonstrated that the engagement of HMGB1 with RAGE promotes the shedding of the receptor and that high levels of soluble forms of RAGE correlate with high levels of chronic ongoing inflammation [42]. Furthermore, circulating sRAGE levels may reflect enhanced tissue RAGE expression in diabetic vasculature [43]. In the present study, we identified a significant positive correlation between the serum levels of sRAGE and the biomarker of endothelial activation and dysfunction sICAM-1 consistent with a previous study [32]. These findings suggest that RAGE signaling pathway is involved in the pathogenesis of endothelial activation 
and dysfunction in diabetes and that these factors may be coregulated in diabetic retinopathy.

sRAGE, a truncated form of the receptor, binds ligands with affinity equal to that of cellular RAGE. It, therefore, has the ability to prevent RAGE signaling acting as a "decoy" by binding ligands and preventing them from reaching the cell surface RAGE. In vitro, sRAGE added to cultured cells blocked the effects of RAGE ligands on expression of inflammatory markers, cellular migration and proliferation, and cytotoxicity [44]. sRAGE has successfully been used in a variety of animal disease models to antagonize RAGE-mediated pathologic processes $[44,45]$. Several studies showed that sRAGE might beneficially impact early vascular and neuronal dysfunction in the diabetic retina. Systemic administration of sRAGE inhibits blood-retinal barrier breakdown, leukostasis, and expression of ICAM-1 in the retina of diabetic animals [46]. In addition, attenuation of the RAGE axis with sRAGE ameliorated retinal neuronal dysfunction and reduced the development of capillary lesions in a murine model of nonproliferative diabetic retinopathy [47]. Our results suggest that elevated levels of sRAGE in the serum from patients with PDR potentially negatively regulate inflammation and that sRAGE is secreted extracellularly as a negative feedback mechanism to limit diabetes-induced retinal vascular and neuronal dysfunction.

Lipid peroxidation is considered a hallmark of oxidative stress. In our study, there was a significant increase in lipid peroxidation in the serum from patients with PDR and in the retinas of diabetic rats as measured by TBARS formation. In addition, intravitreal administration of HMGB1 to normal rats induced significant upregulation of TBARS in the retina. These results are in agreement with several studies that reported elevated levels of circulating TBARS in diabetic subjects [48-50] and that the expression of TBARS is increased in the retinas of diabetic rats [51]. Our analysis showed significant positive correlations between the serum levels of TBARS and sRAGE, sICAM-1, and MCP-1. Our results are consistent with a previous report that demonstrated a significant positive correlation between plasma levels of TBARS and sICAM-1 in patients with type 1 diabetes mellitus [52] and that hyperglycemia-induced oxidative stress induces an increase of circulating ICAM-1 levels [53] and the expression of RAGE in human endothelial cells [54]. On the basis of our findings, we propose a causal relationship linking hyperglycemia, activation of HMGB1/RAGE signaling axis, neuroinflammation, oxidative stress, and diabetic retinal neurodegeneration.

In conclusion, these data suggest that diabetes-induced increased oxidative stress, downregulation of BDNF and synaptophysin, and upregulation of cleaved caspase-3 were also induced by HMGB1. The HMGB1 inhibitor GA attenuated diabetes-induced downregulation of BDNF in the retinas of rats. Collectively, our present data suggest that blocking HMGB1 signaling pathways might be a novel therapeutic strategy for neuronal dysfunction in vision-threatening diabetic retinopathy.

\section{Conflict of Interests}

The authors declare that they have no conflict of interests.

\section{Acknowledgments}

The authors thank Ms. Connie B. Unisa-Marfil for secretarial work. This work was supported by Dr. Nasser Al-Rasheed Research Chair in Ophthalmology (Abu El-Asrar AM).

\section{References}

[1] M. Seki, T. Tanaka, H. Nawa et al., "Involvement of brainderived neurotrophic factor in early retinal neuropathy of streptozotocin-induced diabetes in rats: therapeutic potential of brain-derived neurotrophic factor for dopaminergic amacrine cells," Diabetes, vol. 53, no. 9, pp. 2412-2419, 2004.

[2] A. J. Barber, "A new view of diabetic retinopathy: a neurodegenerative disease of the eye," Progress in NeuroPsychopharmacology and Biological Psychiatry, vol. 27, no. 2, pp. 283-290, 2003.

[3] M. Sasaki, Y. Ozawa, T. Kurihara et al., "Neurodegenerative influence of oxidative stress in the retina of a murine model of diabetes," Diabetologia, vol. 53, no. 5, pp. 971-979, 2010.

[4] A. M. Abu El-Asrar, L. Dralands, L. Missotten, I. A. Al-Jadaan, and K. Geboes, "Expression of apoptosis markers in the retinas of human subjects with diabetes," Investigative Ophthalmology and Visual Science, vol. 45, no. 8, pp. 2760-2766, 2004.

[5] H. Zong, M. Ward, and A. W. Stitt, "AGEs, RAGE, and diabetic retinopathy," Current Diabetes Reports, vol. 11, no. 4, pp. 244252,2011

[6] M. Seki, H. Nawa, T. Fukuchi, H. Abe, and N. Takei, "BDNF is upregulated by postnatal development and visual experience: quantitative and immunohistochemical analyses of BDNF in the rat retina," Investigative Ophthalmology and Visual Science, vol. 44, no. 7, pp. 3211-3218, 2003.

[7] K. R. G. Martin, H. A. Quigley, D. J. Zack et al., "Gene therapy with brain-derived neurotrophic factor as a protection: retinal ganglion cells in a rat glaucoma model," Investigative Ophthalmology and Visual Science, vol. 44, no. 10, pp. 43574365, 2003.

[8] D. K. Binder and H. E. Scharfman, "Brain-derived neurotrophic factor," Growth Factors, vol. 22, no. 3, pp. 123-131, 2004.

[9] J. R. van Beijnum, W. A. Buurman, and A. W. Griffioen, "Convergence and amplification of toll-like receptor (TLR) and receptor for advanced glycation end products (RAGE) signaling pathways via high mobility group B1 (HMGB1)," Angiogenesis, vol. 11, no. 1, pp. 91-99, 2008.

[10] C. J. Treutiger, G. E. Mullins, A. S. M. Johansson et al., "High mobility group 1 B-box mediates activation of human endothelium," Journal of Internal Medicine, vol. 254, no. 4, pp. 375-385, 2003.

[11] C. Fiuza, M. Bustin, S. Talwar et al., "Inflammation-promoting activity of HMGB1 on human microvascular endothelial cells," Blood, vol. 101, no. 7, pp. 2652-2660, 2003.

[12] Z. G. Luan, H. Zhang, P. T. Yang, X. C. Ma, C. Zhang, and R. X. Guo, "HMGB1 activates nuclear factor- $\kappa$ B signaling by RAGE and increases the production of TNF- $\alpha$ in human umbilical vein endothelial cells," Immunobiology, vol. 215, no. 12, pp. 956-962, 2010.

[13] M. Takeuchi, J. I. Takino, and S. I. Yamagishi, "Involvement of the toxic AGEs (TAGE)-RAGE system in the pathogenesis of diabetic vascular complications: a novel therapeutic strategy," Current Drug Targets, vol. 11, no. 11, pp. 1468-1482, 2010.

[14] S. F. Yan, S. D. Yan, R. Ramasamy, and A. M. Schmidt, "Tempering the wrath of RAGE: an emerging therapeutic 
strategy against diabetic complications, neurodegeneration, and inflammation," Annals of Medicine, vol. 41, no. 6, pp. 408422, 2009.

[15] H. M. Gao, H. Zhou, F. Zhang, B. C. Wilson, W. Kam, and J. S. Hong, "HMGB1 acts on microglia Macl to mediate chronic neuroinflammation that drives progressive neurodegeneration," Journal of Neuroscience, vol. 31, no. 3, pp. 1081-1092, 2011.

[16] A. M. Joussen, V. Poulaki, M. L. Le et al., "A central role for inflammation in the pathogenesis of diabetic retinopathy," The FASEB Journal, vol. 18, no. 12, pp. 1450-1452, 2004.

[17] K. Miyamoto, S. Khosrof, S. E. Bursell et al., "Prevention of leukostasis and vascular leakage in streptozotocin-induced diabetic retinopathy via intercellular adhesion molecule-1 inhibition," Proceedings of the National Academy of Sciences of the United States of America, vol. 96, no. 19, pp. 10836-10841, 1999.

[18] J. B. Kim, S. C. Joon, Y. M. Yu et al., "HMGB1, a novel cytokinelike mediator linking acute neuronal death and delayed neuroinflammation in the postischemic brain," Journal of Neuroscience, vol. 26, no. 24, pp. 6413-6421, 2006.

[19] I. D. Kim, J. H. Shin, S. W. Kim et al., "Intranasal delivery of HMGB1 siRNA confers target gene knockdown and robust neuroprotection in the postischemic brain," Molecular Therapy, vol. 20, pp. 829-839, 2012.

[20] S. W. Kim, Y. Jin, J. H. Shin et al., "Glycyrrhizic acid affords robust neuroprotection in the postischemic brain via antiinflammatory effect by inhibiting HMGB1 phosphorylation and secretion," Neurobiology of Disease, vol. 46, no. 1, pp. 147-156, 2012.

[21] G. Dvoriantchikova, E. Hernandez, J. Grant, A. R. Santos, H. Yang, and D. Ivanvov, "The high-mobility group box-1 nuclear factor mediates retinal injury after ischemia reperfusion," Investigative Ophthalmology \& Visual Science, vol. 52, pp. 7187-7194, 2011.

[22] A. M. Abu El-Asrar, M. I. Nawaz, D. Kangave, M. M. Siddiquei, and K. Geboes, "Osteopontin and other regulators of angiogenesis and fibrogenesis in the vitreous from patients with proliferative vitreoretinal disorders," Mediators of Inflammation, vol. 2012, Article ID 493043, 8 pages, 2012.

[23] A. M. Abu El-Asrar, M. I. Nawaz, D. Kangave et al., "Highmobility group box-1 and biomarkers of inflammation in the vitreous from patients with proliferative diabetic retinopathy," Molecular Vision, vol. 17, pp. 1829-1838, 2011.

[24] A. M. Abu El-Asrar, L. Missotten, and K. Geboes, "Expression of high-mobility groups box-1/receptor for advanced glycation end products/osteopontin/early growth response-1 pathway in proliferative vitreoretinal epiretinal membranes," Molecular Vision, vol. 17, pp. 508-518, 2011.

[25] L. Mollica, F. de Marchis, A. Spitaleri et al., "Glycyrrhizin binds to high-mobility group box 1 protein and inhibits its cytokine activities," Chemistry \& Biology, vol. 14, pp. 431-441, 2007.

[26] G. Mohammad, M. M. Siddiquei, A. Othman, M. Al-Shabrawey, and A. M. Abu El-Asrar, "High-mobility group box-1 protein activates inflammatory signaling pathway components and disrupts retinal vascular-barrier in the diabetic retina," Experimental Eye Research, vol. 107, pp. 101-109, 2013.

[27] H. S. Phillips, J. M. Hains, M. Armanini, G. R. Laramee, S. A. Johnson, and J. W. Winslow, "BDNF mRNA is decreased in the hippocampus of individuals with Alzheimer's disease," Neuron, vol. 7, no. 5, pp. 695-702, 1991.

[28] M. Yamanaka, Y. Itakura, M. Ono-Kishino, A. Tsuchida, T. Nakagawa, and M. Taiji, "Intermittent administration of brain-derived neurotrophic factor (BDNF) ameliorates glucose metabolism and prevents pancreatic exhaustion in diabetic mice," Journal of Bioscience and Bioengineering, vol. 105, no. 4, pp. 395-402, 2008.

[29] M. Yamanaka, Y. Itakura, A. Tsuchida, T. Nakagawa, H. Noguchi, and M. Taiji, "Comparison of the antidiabetic effects of brain-derived neurotrophic factor and thiazolidinediones in obese diabetic mice," Diabetes, Obesity and Metabolism, vol. 9, no. 6, pp. 879-888, 2007.

[30] K. S. Krabbe, A. R. Nielsen, R. Krogh-Madsen et al., "Brainderived neurotrophic factor (BDNF) and type 2 diabetes," Diabetologia, vol. 50, no. 2, pp. 431-438, 2007.

[31] A. Fujinami, K. Ohta, H. Obayashi et al., "Serum brain-derived neurotrophic factor in patients with type 2 diabetes mellitus: relationship to glucose metabolism and biomarkers of insulin resistance," Clinical Biochemistry, vol. 41, no. 10-11, pp. 812-817, 2008.

[32] J. Skrha Jr., M. Kalousovā, J. Svarcovā et al., "Relationship of soluble RAGE and RAGE ligands HMGB1 and EN-RAGE to endothelial dysfunction in type 1 and type 2 diabetes mellitus," Experimental and Clinical Endocrinology \& Diabetes, vol. 120, pp. 277-281, 2012.

[33] J. W. M. Nin, I. Ferreira, C. G. Schalkwijk et al., "Levels of soluble receptor for AGE are cross-sectionally associated with cardiovascular disease in type 1 diabetes, and this association is partially mediated by endothelial and renal dysfunction and by low-grade inflammation: the EURODIAB Prospective Complications Study," Diabetologia, vol. 52, no. 4, pp. 705-714, 2009.

[34] I. Spiwoks-Becker, L. Vollrath, M. W. Seeliger, G. Jaissle, L. G. Eshkind, and R. E. Leube, "Synaptic vesicle alterations in rod photoreceptors of synaptophysin-deficient mice," Neuroscience, vol. 107, no. 1, pp. 127-142, 2001.

[35] H. D. VanGuilder, R. M. Brucklacher, K. Patel, R. W. Ellis, W. M. Freeman, and A. J. Barber, "Diabetes downregulates presynaptic proteins and reduces basal synapsin i phosphorylation in rat retina," European Journal of Neuroscience, vol. 28, no. 1, pp. 111, 2008.

[36] T. Kurihara, Y. Ozawa, N. Nagai et al., "Angiotensin II type 1 receptor signaling contributes to synaptophysin degradation and neuronal dysfunction in the diabetic retina," Diabetes, vol. 57, no. 8, pp. 2191-2198, 2008.

[37] M. Challier, S. Jacqueminet, O. Benabdesselam, A. Grimaldi, and J. L. Beaudeux, "Increased serum concentrations of soluble receptor for advanced glycation endproducts in patients with type 1 diabetes," Clinical Chemistry, vol. 51, no. 9, pp. 1749-1750, 2005.

[38] K. C. Tan, S. W. Shiu, W. S. Chow, L. Leng, R. Bucala, and D. J. Betteridge, "Association between serum levels of soluble receptor for advanced glycation end products and circulating advanced glycation end products in type 2 diabetes," Diabetologia, vol. 49, no. 11, pp. 2756-2762, 2006.

[39] M. Nowak, T. Wielkoszyński, B. Marek et al., "Blood serum levels of vascular cell adhesion molecule (sVCAM-1), intercellular adhesion molecule (sICAM-1) and endothelial leucocyte adhesion molecule-1 (ELAM-1) in diabetic retinopathy," Clinical and Experimental Medicine, vol. 8, no. 3, pp. 159-164, 2008.

[40] S. Kado and N. Nagata, "Circulating intercellular adhesion molecule-1, vascular cell adhesion molecule-1, and E-selectin in patients with type 2 diabetes mellitus," Diabetes Research and Clinical Practice, vol. 46, no. 2, pp. 143-148, 1999. 
[41] M. N. Pham, M. I. Hawa, M. Roden et al., "Increased serum concentrations of adhesion molecules but not of chemokines in patients with type 2 diabetes compared with patients with type 1 diabetes and latent autoimmune diabetes in adult age: action LADA5," Diabetic Medicine, vol. 29, no. 4, pp. 470-478, 2012.

[42] A. Raucci, S. Cugusi, A. Antonelli et al., "A soluble form of the receptor for advanced glycation endproducts (RAGE) is produced by proteolytic cleavage of the membrane-bound form by the sheddase a disintegrin and metalloprotease 10 (ADAM10)," The FASEB Journal, vol. 22, no. 10, pp. 3716-3727, 2008.

[43] K. Nakamura, S. I. Yamagishi, H. Adachi et al., "Serum levels of sRAGE, the soluble form of receptor for advanced glycation end products, are associated with inflammatory markers in patients with type 2 diabetes," Molecular Medicine, vol. 13, no. 3-4, pp. 185-189, 2007.

[44] S. F. Yan, R. Ramasamy, and A. M. Schmidt, "Soluble RAGE: therapy and biomarker in unraveling the RAGE axis in chronic disease and aging," Biochemical Pharmacology, vol. 79, no. 10, pp. 1379-1386, 2010.

[45] H. Maillard-Lefebvre, E. Boulanger, M. Daroux, C. Gaxatte, B. I. Hudson, and M. Lambert, "Soluble receptor for advanced glycation end products: a new biomarker in diagnosis and prognosis of chronic inflammatory diseases," Rheumatology, vol. 48, no. 10, pp. 1190-1196, 2009.

[46] Y. Kaji, T. Usui, S. Ishida et al., "Inhibition of diabetic leukostasis and blood-retinal barrier breakdown with a soluble form of a receptor for advanced glycation end products," Investigative Ophthalmology and Visual Science, vol. 48, no. 2, pp. 858-865, 2007.

[47] G. R. Barile, S. I. Pachydaki, S. R. Tari et al., "The RAGE axis in early diabetic retinopathy," Investigative Ophthalmology and Visual Science, vol. 46, no. 8, pp. 2916-2924, 2005.

[48] S. Stranges, J. M. Dorn, R. P. Donahue et al., "Oxidation, type 2 diabetes, and coronary heart disease: a complex interaction: findings from a population-based study," Diabetes Care, vol. 31, no. 9, pp. 1864-1866, 2008.

[49] H. M. Turk, A. Sevinc, C. Camci et al., "Plasma lipid peroxidation products and antioxidant enzyme activities in patients with type 2 diabetes mellitus," Acta Diabetologica, vol. 39, no. 3, pp. 117-122, 2002.

[50] M. E. Hartnett, R. D. Stratton, R. W. Browne, B. A. Rosner, R. J. Lanham, and D. Armstrong, "Serum markers of oxidative stress and severity of diabetic retinopathy," Diabetes Care, vol. 23, no. 2, pp. 234-240, 2000.

[51] R. A. Kowluru, "Diabetes-induced elevations in retinal oxidative stress, protein kinase $\mathrm{C}$ and nitric oxide are interrelated," Acta Diabetologica, vol. 38, no. 4, pp. 179-185, 2001.

[52] M. Lechleitner, T. Koch, M. Herold, A. Dzien, and F. Hoppichler, "Tumour necrosis factor-alpha plasma level in patients with type 1 diabetes mellitus and its association with glycaemic control and cardiovascular risk factors," Journal of Internal Medicine, vol. 248, no. 1, pp. 67-76, 2000.

[53] A. Ceriello, E. Falleti, E. Motz et al., "Hyperglycemia-induced circulating ICAM-1 increase in diabetes mellitus: the possible role of oxidative stress," Hormone and Metabolic Research, vol. 30, no. 3, pp. 146-149, 1998.

[54] D. Yao and M. Brownlee, "Hyperglycemia-induced reactive oxygen species increase expression of the receptor for advanced glycation end products (RAGE) and RAGE ligands," Diabetes, vol. 59, no. 1, pp. 249-255, 2010. 


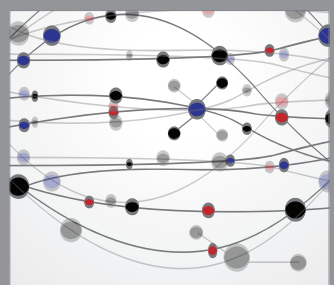

The Scientific World Journal
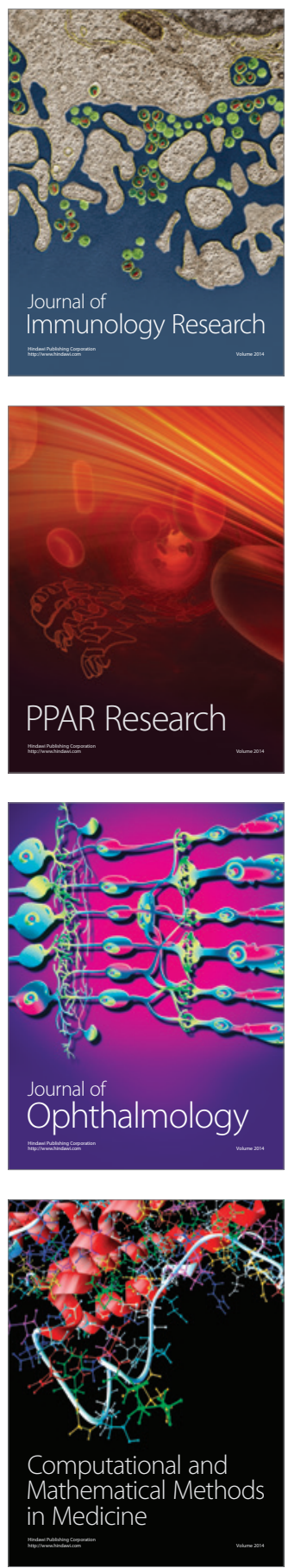

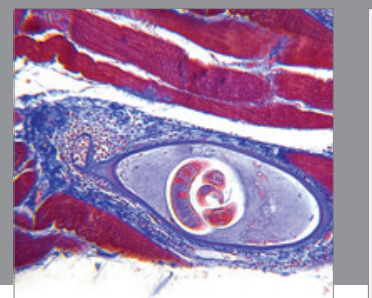

Gastroenterology

Research and Practice
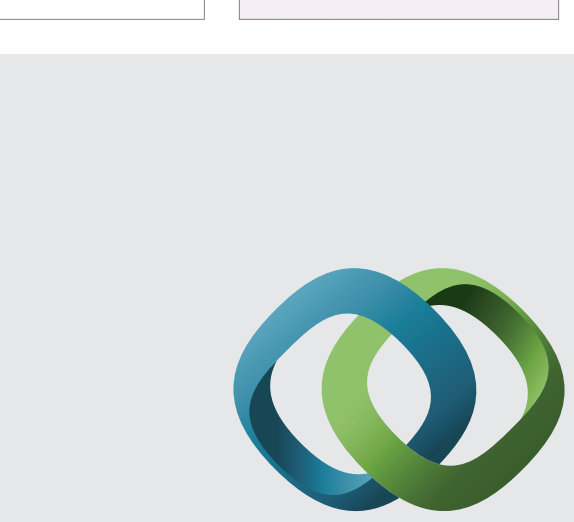

\section{Hindawi}

Submit your manuscripts at

http://www.hindawi.com
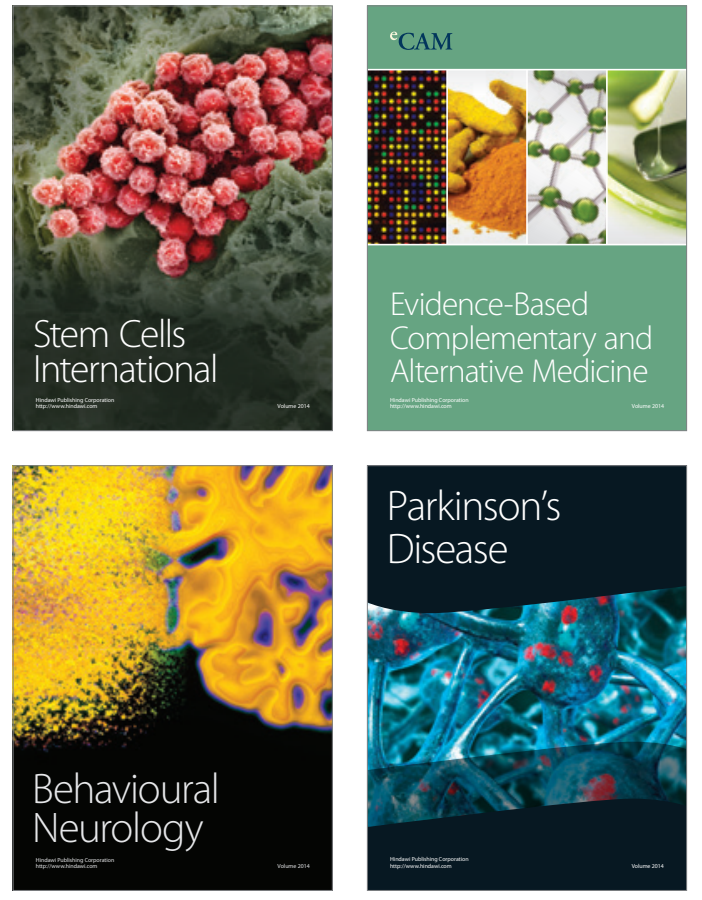
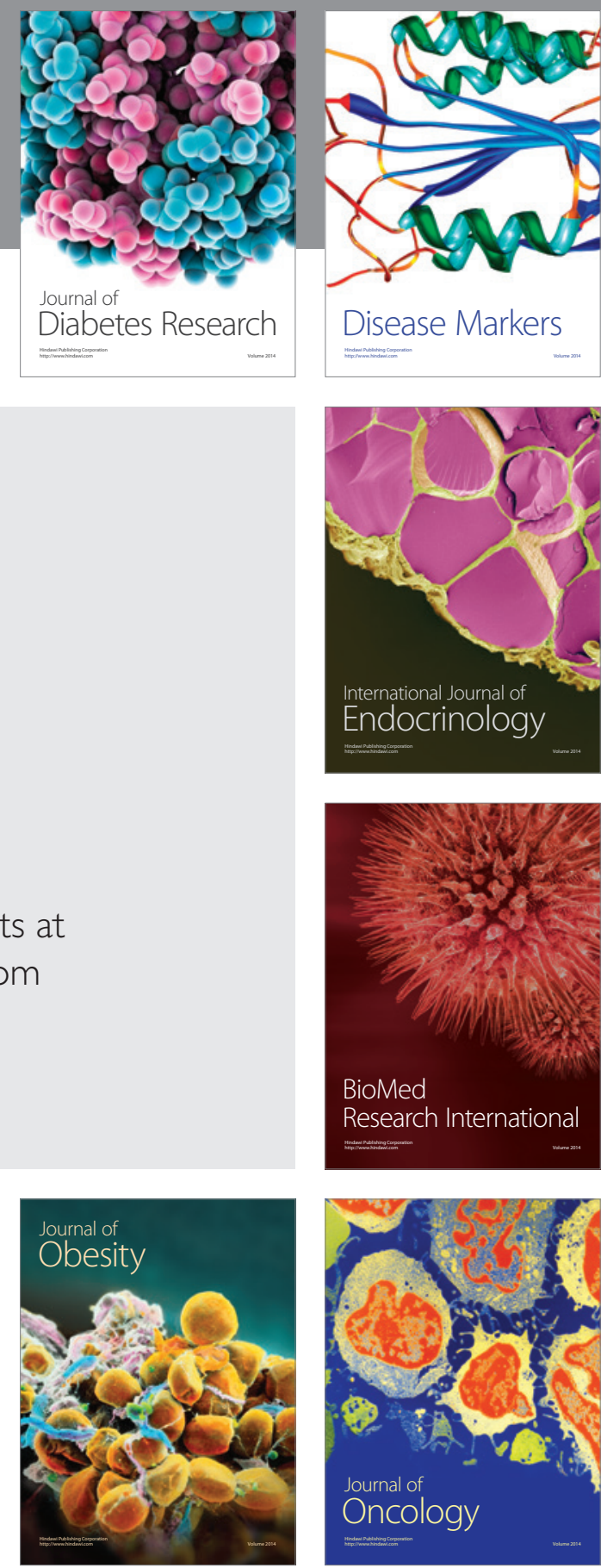

Disease Markers
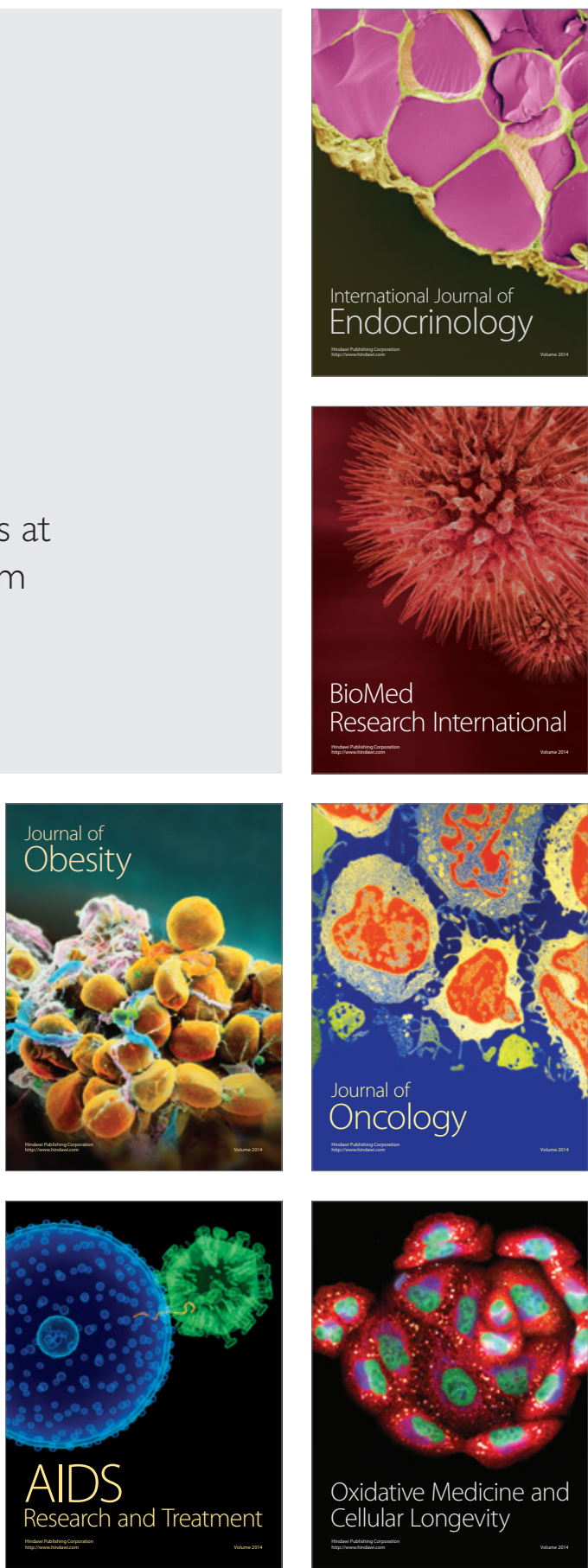